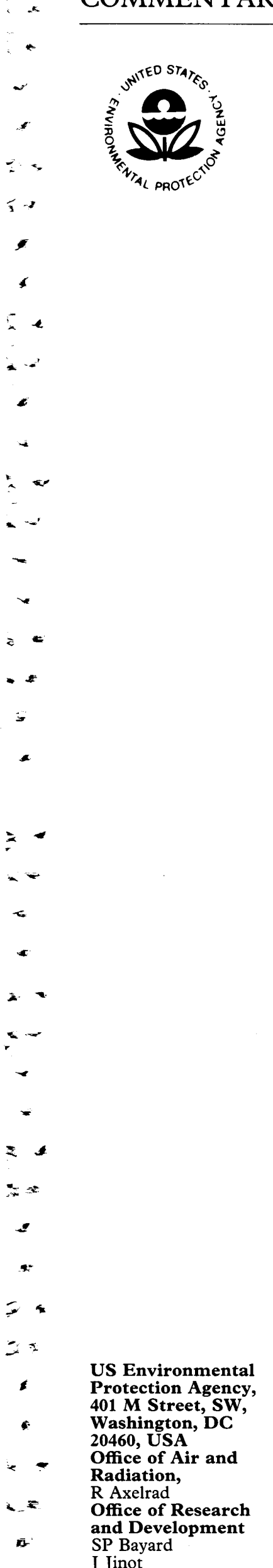

\title{
Setting the record straight: secondhand smoke is a preventable health risk
}

\author{
Robert Axelrad, Steven P Bayard, Jennifer Jinot
}

In Fanuary 1993 the US Environmental Protection Agency (EPA) released its report Respiratory health effects of passive smoking: lung cancer and other disorders. The report represents the most comprehensive and up-todate review of the evidence on the respiratory health effects of passive smoking. An executive summary of the report was published in Tobacco Control (1993; 2: 71-7). The tobacco industry, perhaps fearful of the impact of the report on the adoption of clean indoor air policies, has sought to attack it through litigation and a massive advertising and public relations campaign. The authors of this commentary have prepared a spirited defence of the EPA report and a rebuttal to the industry's arguments. Their paper was approved and released by the EPA in fune 1994 as an official agency document, and is reproduced below. Several other articles related to the EPA report, and the industry's response to it, have been published previously in Tobacco Control (1992; 1: 166-7; 1992; 1: 208-19; 1993; 2: 3-4; and 1993; 2 : 103-13). - ED

\section{Introduction}

In early 1993, EPA released a report (Respiratory health effects of passive smoking: lung cancer and other disorders; EPA/600/6$90 / 006 \mathrm{~F}$ ) that evaluated the respiratory health effects from breathing secondhand smoke (also called environmental tobacco smoke). In that report, EPA concluded that secondhand smoke causes lung cancer in adult nonsmokers and impairs the respiratory health of children. These findings are very similar to ones made previously by the National Academy of Sciences and the US Surgeon General.

The EPA report classified secondhand smoke as a Group A carcinogen, a designation which means that there is sufficient evidence that the substance causes cancer in humans. The Group A designation has been used by EPA for only 15 other pollutants, including asbestos, radon, and benzene. Only secondhand smoke has actually been shown in studies to cause cancer at typical environmental levels. EPA estimates that approximately 3000 American nonsmokers die each year from lung cancer caused by secondhand smoke.

Every year, an estimated 150000 to 300000 children under 18 months of age get pneumonia or bronchitis from breathing secondhand tobacco smoke. Secondhand smoke is a risk factor for the development of asthma in children and worsens the condition of up to one million asthmatic children.

EPA has clear authority to inform the public about indoor air pollution health risks and what can be done to reduce those risks. EPA has a particular responsibility to do everything possible to warn of risks to the health of children.

A recent high profile advertising and public relations campaign by the tobacco industry may confuse the American public about the risks of secondhand smoke. EPA believes it's time to set the record straight about an indisputable fact: secondhand smoke is a real and preventable health risk.

EPA absolutely stands by its scientific and well documented report. The report was the subject of an extensive open review both by the public and by EPA's Science Advisory Board (SAB), a panel of independent scientific experts. Virtually every one of the arguments about lung cancer advanced by the tobacco industry and its consultants was addressed by the SAB. The panel concurred in the methodology and unanimously endorsed the conclusions of the final report.

The report has also been endorsed by the US Department of Health and Human Services, the National Cancer Institute, the Surgeon General, and many major health organizations.

Classification of secondhand smoke as a known human (group A) carcinogen

The finding that secondhand smoke causes lung cancer in nonsmoking adults is based on the total weight of the available evidence and is not dependent on any single analysis. This evidence includes several important facts.

First, it is indisputable that smoking tobacco causes lung cancer in humans, and there is no evidence that there is a threshold below which smoking will not cause cancer.

Second, although secondhand smoke is a dilute mixture of "mainstream" smoke exhaled by smokers and "sidestream" smoke from the burning end of a cigarette or other tobacco product, it is chemically similar to the smoke inhaled by smokers, and contains a number of carcinogenic compounds.

Third, there is considerable evidence that large numbers of people who do not smoke are 
exposed to, absorb, and metabolize significant amounts of secondhand smoke.

Fourth, there is supporting evidence from laboratory studies of the ability of secondhand smoke both to cause cancer in animals and to damage DNA, which is recognized by scientists as being an instrumental mechanism in cancer develcpment.

Finally, EPA conducted multiple analyses on the then-available 30 epidemiology studies from eight different countries which examined the association between secondhand smoke and lung cancer in women who never smoked themselves but were exposed to their husband's smoke. Since the epidemiology studies are the major thrust of the tobacco industry arguments against the EPA report, these studies are examined in more detail below.

\section{The epidemiology studies}

The most important aspect of the review of the epidemiology studies is the remarkable consistency of results across studies that support a causal association between secondhand smoke and lung cancer.

In assessing the studies in several different ways, it becomes clear that the extent of the consistency defies attribution to chance. When looking only at the simple measure of exposure of whether the husband ever smoked, 24 of 30 studies reported an increase in risk for nonsmoking women with smoking husbands. Since many of these studies were small, the chance of declaring these increases statistically significant was small. Still, nine of these were statistically significant, and the probability that this many of the studies would be statistically significant merely by chance is less than 1 in 10 thousand.
The simple overall comparison of risks in ever $v s$ never exposed to spousal smoking tends to hide true increases in risk in two ways. First, it categorizes many women as never exposed who actually received exposure from sources other than spousal smoking. It also includes some women as exposed who actually received little exposure from their husband's smoking. One way to correct for this latter case is to look at the women whose husbands smoked the most. When one looks at the 17 studies that examined cancer effects based on the level of exposure of the subjects, every study found an increased lung cancer risk among those subjects who were most exposed. Nine were statistically significant. The probability of 9 out of 17 studies showing statistically significant results occurring by chance is less than 1 in ten million.

Probably the most important finding for a causal relationship is one of increasing response with increasing exposure, since such associations cannot usually be explained by other factors. Such exposure-response trends were seen in all 14 studies that examined the relationship between level of exposure and effect. In 10 of the studies the trends were statistically significant. The probability of this happening by chance is less than 1 in a billion.

It is unprecedented for such a consistency of results to be seen in epidemiology studies of cancer from environmental levels of a pollutant. One reason is that it is extremely difficult to detect an effect when virtually everyone is exposed, as is the case with secondhand smoke. However, consistent increased risks for those most exposed and consistent trends of increasing exposure showing an increasing effect provide strong evidence that secondhand smoke increases the risk of lung cancer in nonsmokers. 
30 EPIDEMIOLOGY STUDIES OF ETS AND LUNG CANCER

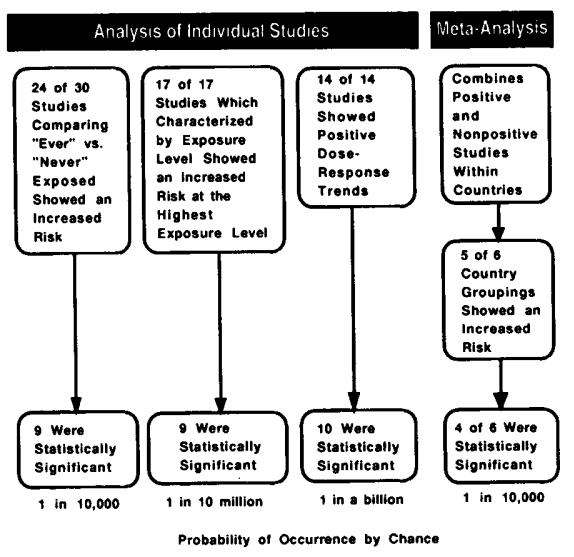

Figure 230 epidemiology studies of ETS and lung cancer

How big a lung cancer risk for adults?

The evidence is clear and consistent: secondhand smoke is a cause of lung cancer in adults who don't smoke. EPA has never claimed that minimal exposure to secondhand smoke poses a huge individual cancer risk. Even though the lung cancer risk from secondhand smoke is relatively small compared to the risk from direct smoking, unlike a smoker who chooses to smoke, the nonsmoker's risk is often involuntary. In addition, exposure to secondhand smoke varies tremendously among exposed individuals. For those who must live or work in close proximity to one or more smokers, the risk would certainly be greater than for those less exposed.

EPA estimates that secondhand smoke is responsible for about 3000 lung cancer deaths each year among nonsmokers in the US; of these, the estimate is 800 from exposure to secondhand smoke at home and 2200 from exposure in work or social situations.

\section{The risks to children are widely acknowledged}

The conclusion that secondhand smoke causes respiratory effects in children is widely shared and virtually undisputed. Even the tobacco industry does not contest these effects in its media and public relations campaign.

EPA estimates that, every year, between 150000 and 300000 children under $1-1 / 2$ years of age get bronchitis or pneumonia from breathing secondhand tobacco smoke, resulting in thousands of hospitalizations. In children under 18 years of age, secondhand smoke exposure also results in more coughing and wheezing, a small but significant decrease in lung function, and an increase in fluid in the middle ear. Children with asthma have more frequent and more severe asthma attacks because of exposure to secondhand smoke, which is also a risk factor for the onset of asthma in children who did not previously have symptoms.
Other risks

Secondhand smoke contains strong irritants and sensitizers and many adults, as well as children, suffer irritation and other acute effects whenever they are exposed to secondhand smoke. In addition, there is mounting evidence that exposure to secondhand smoke can have an effect on the cardiovascular system, although the EPA report does not address this issue.

\section{Tobacco industry media campaign}

The tobacco industry is raising numerous issues which may distract the public from the fact that secondhand smoke poses a real and preventable health risk. The tobacco industry neither acknowledges nor disputes EPA's conclusions of respiratory effects in children. It focuses instead on EPA's findings on lung cancer.

The overall thrusts of the tobacco industry's arguments are that EPA manipulated the lung cancer data to come to a predetermined conclusion. The industry also argues that a nonsmoker's exposure to secondhand smoke is so small as to be insignificant. The argument on minimal exposure is belied both by the acute irritation and respiratory effects and the fallacy of the "cigarette equivalents" approach discussed below. Responses to the specific criticisms of EPA's assessment of the lung cancer data follow.

\section{THE 11 US LUNG CANCER STUDIES}

Critics of the EPA report argue that by normal statistical standards, none of the 11 US studies included in the EPA report showed a statistically significant increase in the simple overall risk measure, and that EPA should therefore have been unable to conclude that secondhand smoke causes lung cancer in nonsmokers. These critics are misrepresenting a small part of the total evidence on secondhand smoke and lung cancer.

The consistency of study results in the highest exposure category and exposure-response trends discussed above also apply to the US studies. For example, seven of the 11 US studies had fewer than 45 cases, making statistical comparisons difficult. Nonetheless, eight of the 11 had increased overall risks, and for the seven studies which reported on risks by amount of exposure, the highest exposure groups in all seven had increased risks. While the 11 US studies are not, by themselves, conclusive, they do support the conclusion that secondhand smoke is causally associated with lung cancer.

STUDIES COMPLETED SINCE RELEASE OF THE EPA REPORT

Critics claim that had EPA not "excluded" the recent Brownson study, the Agency could not have concluded that secondhand smoke causes cancer. In fact, four new lung cancer 
epidemiology studies, including the Brownson study, have been published since the literature review cutoff date for the 1993 EPA report, and all support EPA's conclusions. Three of these are large US studies funded, at least in part, by the National Cancer Institute. A 1992 study of Florida women by Stockwell et al found a $60 \%$ overall increased risk of lung cancer from exposure to their husband's smoke, with significant results for both the highest exposure group and the exposureresponse trend. The 1992 study of Missouri women by Brownson et al found no overall increased risk, but did demonstrate a significant increase in risk in the highest spousal smoking exposure group and a positive exposure-response trend.

The 1994 study by Fontham et al of women in two California and three Southern cities is the largest case-control study on the subject ever conducted and is considered by EPA to be the best designed study on secondhand smoke and lung cancer conducted to date. This study found significantly increased risks for overall exposure and in the highest exposure group and a strong positive exposure-response relationship. These findings were significant not only for exposure from spouses, but also for exposure in the workplace and in social situations.

\section{$90 \%$ VS $95 \%$ CONFIDENCE INTERVALS}

Critics of the EPA report have charged that EPA changed the confidence interval in order to come to a predetermined conclusion. However, the conclusion that secondhand smoke is a known human carcinogen simply does not hinge on whether or not a $95 \%$ or $90 \%$ "confidence interval" was used. A confidence interval is used to display variability in relative risk estimates in the epidemiology studies. As discussed above, the group A designation is based on the total weight of the available evidence. The consistency of results that are seen in the numerous studies examined lead to a certainty of greater than $99.9 \%$ that secondhand smoke increases the risk of lung cancer in nonsmokers.

Use of what is called in statistics a "onetailed test of significance," which often corresponds to a $90 \%$ confidence interval, is a standard and appropriate statistical procedure in certain circumstances. The "one-tailed test" is used when there is prior evidence that if there is an effect from a substance, it is highly likely to be an adverse rather than a protective effect, or vice versa. In the case of secondhand smoke, an extensive database exists for direct smoking indicating that if chemically similar secondhand smoke also has a lung cancer effect, this effect is likely to be similarly adverse. EPA used one-tailed significance tests for lung cancer in both external drafts of the risk assessment document as well as the final report. Ninety percent confidence intervals were also used in other EPA cancer risk assessments, including methylene chloride, coke oven emissions, radon, nickel, and dioxin.
In the non-cancer respiratory effects portions of the report, "two-tailed tests" and $95 \%$ confidence intervals were used, since there was less prior evidence from smokers to suggest that secondhand smoke would cause bronchitis, pneumonia, and ear infections in children.

\section{THE META-ANALYSIS}

Meta-analysis was used for the lung cancer data as an objective method of combining results from many studies and was specifically endorsed by the SAB for use with this database. Some critics argue both that the meta-analysis was not an appropriate technique, and that had EPA included the Brownson study (addressed above) in the metaanalysis of overall spousal exposure, EPA could not possibly have classified secondhand smoke as a known human carcinogen. This just isn't true.

The finding that secondhand smoke is a known cause of lung cancer in humans is based on all the evidence and is not dependent on the meta-analysis of the simple ever- $v s$ neverexposed comparisons, as the critics suggest. If the meta-analysis were removed from the report entirely, the findings would be precisely the same. The meta-analysis was used primarily for estimating and quantifying the population risks from exposure to secondhand smoke, and an alternative approach also used in the report gave very similar results.

\section{CONFOUNDERS}

In the secondhand smoke report, a confounder would be a specific factor that could be responsible for the lung cancer increases observed in nonsmokers instead of secondhand smoke. The tobacco industry and its consultants have suggested, for example, that nonsmoking wives might share in the same poor dietary habits as their smoking husbands, increasing their risk.

The consistency of results across different countries where lifestyle factors, including diet, vary, argues against confounding. For example, while the tobacco industry theorizes that a high fat diet is a confounding factor, the studies from Japan, where dietary fat intake is among the lowest in the world, show a strong dose-response relationship for secondhand smoke and lung cancer.

The EPA report did examine the available data for six potential confounders such as occupation, dietary factors, and history of lung disease, and concluded that none was likely to explain the lung cancer increases seen in the studies.

The 1994 Fontham et al study controlled for diet and other potential confounders, and concluded, "These observations indicate that the strong association in this study between adult secondhand smoke exposure and lung cancer risk cannot be attributed to any likely confounder." 
THE "THRESHOLD THEORY"

Although some have argued that tobacco smoke cannot cause cancer below a certain level, there is no evidence that this threshold exists. In the absence of such evidence, carcinogens at any level are considered by EPA to increase risk somewhat, although the degree of risk certainly is reduced as exposure decreases. The increased risks observed in the secondhand smoke epidemiology studies are further evidence that any threshold for secondhand smoke would have to be at very low levels.

4

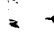

$-\infty$

$=$

$*$

$: \cdots$

$-\infty$

$=$

$\therefore$

- $s$

3

\section{"CIGARETTE EQUIVALENTS"}

The tobacco industry uses the "cigarette equivalent" method of comparing smokers' and nonsmokers' exposures to a single component of tobacco smoke to infer that a nonsmoker's exposure to tobacco smoke is insignificant. However, the cigarette equivalent method has no scientific support, and was rejected by the $S A B$ panel that reviewed the EPA report. Among the many problems with this method is the fact that while secondhand smoke and mainstream smoke contain the same approximately 4000 compounds, their ratios of individual compounds differ by factors in the thousands. Thus, there is no single compound in tobacco smoke that is an adequate indicator for drawing such comparisons. An RJ Reynolds newspaper ad, while utilizing the method, acknowledges it may not be relevant for assessing risk from secondhand smoke.

\section{RESIDENTIAL EXPOSURES TRANSLATED TO THE WORKPLACE}

The tobacco industry frequently argues that because most studies were based on residential exposures, secondhand smoke has not been shown to be a hazard in the workplace. A substance capable of causing cancer in one environment is certainly capable of causing it in any other environment where exposures are comparable, as is the case with residential and workplace exposure to secondhand smoke. In fact, the 1994 Fontham study found a slightly higher risk for workplace exposure than for residential exposures.

THE CONGRESSIONAL RESEARCH SERVICE REPORT The RJ Reynolds' media campaign cites a report prepared by the Congressional Research Service (CRS) on cigarette taxes to fund health care reform to argue that CRS believes that the epidemiological evidence on secondhand smoke and health effects is "weak and uncertain." However, CRS has not taken a position on either EPA's risk assessment or the health effects of passive smoking.
Two economists from CRS, citing material largely prepared by the tobacco industry, included a discussion of EPA's risk assessment in an economic analysis of a cigarette excise tax proposal to fund health care reform. In EPA's view, the CRS economists' cursory look at the issues is not comparable to the exhaustive analyses and rigorous review process which EPA undertook when examining the extensive database on secondhand smoke and respiratory health. EPA is confident that a comprehensive analysis of the secondhand smoke database by expert scientists from CRS, with adequate peer review, will come to conclusions about the risks of secondhand smoke similar to those of EPA and many other organizations.

\section{Cigarette prohibition}

The claim that the government is attempting to bring back prohibition - this time for cigarettes - is a complete fabrication and utter nonsense. EPA's interest is to provide information to protect the nonsmoker from involuntary exposure to a hazardous substance. Having a choice to take a risk for themselves should not permit smokers to impose a risk on others.

\section{Secondhand smoke legislation}

Congress has recently passed, and President Clinton has signed into law, legislation restricting smoking in nearly all public places where federal assistance is provided for services to children. Children exposed to secondhand smoke almost never have a choice. Protecting children from the health effects of secondhand smoke should be a priority for everyone.

The Clinton Administration supports pending legislation (HR 3434, S 1680, S 262) that would protect nonsmokers, including children, from secondhand smoke in most public places. These bills would not take away the smoker's freedom to choose to smoke, nor would it bring government regulation into the home.

The bills would also make good economic sense. EPA estimates that smoking restrictions would result in saving $\$ 4$ billion to $\$ 8$ billion per year in housekeeping and maintenance expenses.

Perhaps most importantly, the bills would prevent thousands of premature deaths of nonsmokers per year and reduce the incidence of respiratory illness in children.

\section{Further information}

For additional information on secondhand smoke and other indoor air pollutants, call EPA's Indoor Air Quality Information Clearinghouse at (1 800)438 4318 (US only) or (1 $301) 5859020$, or fax to $(1301) 5883408$. 